\title{
Guided bone regeneration in peri-implant defects using a 1:1 mixture of cancellous and cortical freeze-dried bone allograft: A randomized controlled trial
}

Won-Pyo Lee, Do-Young Park, Ki-Won Lee, Keon-Il Yang, Byung-Ock Kim, and Sang-Joun Yu*

Department of Periodontology, School of Dentistry, Chosun University, Gwangju, Republic of Korea

\begin{abstract}
The results of guided bone regeneration (GBR) in peri-implant defects using anorganic bovine bone (ABB) were compared with those using a 1:1 mixture of cancellous and cortical freeze-dried bone allograft (FDBA). In total, 37 participants (10 males and 27 females) and 63 sites were evaluated. Full mucoperiosteal flap was reflected followed by implant insertion. The length and width of the defect were measured using a periodontal probe. Furthermore, the most buccal $\left(\mathrm{D}_{\mathrm{is}}\right)$ and lingual $\left(\mathrm{D}_{\mathrm{d}}\right)$ points of the exposed implant surface at the implant shoulder level were determined. The participants were randomly divided into two groups based on the graft material used: $\mathrm{ABB}$ only (control group) and 1:1 mixture of cancellous and cortical FDBA allograft (experimental group). Each transplanted site was covered by the collagen barrier membrane. After 5-6 months of surgery, re-entry was performed, and any residual defect length and width were measured. Moreover, the amount of regenerated bone was measured by calculating the distance from the $D_{i s}$ and $D_{d}$ points to the regenerated bone in the buccolingual direction. Between-group comparisons were performed using the t-test. No differences in defect length, exposed implant surface, and horizontal bone gain were observed between ABB and allograft. Similarly, no significant differences in these measures and the defect width were observed between the two materials in both the maxilla and mandible. The 1:1 mixture of cancellous and cortical FDBA allograft combined with resorbable barrier membrane could be an effective alternative for $\mathrm{ABB}$ for the treatment of peri-implant defects when using GBR.
\end{abstract}

Key Words: Bone regeneration, Bone substitutes, Clinical trial

(c) This is an open-access article distributed under the terms of the Creative Commons Attribution Non-Commercial License (http://creativecommons.org/licenses/by-nc/4.0) which permits unrestricted noncommercial use, distribution, and reproduction in any medium, provided the original work is properly cited.

\section{INTRODUCTION}

Prosthetic restoration using an implant in the partially edentulous or edentulous area has been the most common treatment method [1]. It is necessary to install the implant in the ideal position and direction for it to function properly [2]. If the width of the alveolar crest is insufficient, it is likely that the surface of the dental implant can be exposed after installation, resulting in the failure of the procedure [3]. To avoid this complication, the procedure of bone augmentation is required. Guided bone regeneration (GBR) technique has been applied to augment the defect not only before the installation of dental implant but also at the same time of installation [4]. In particular, it has been noted that the treatment time can be shortened significantly by installing the dental implant using the GBR technique.

Received October 21, 2019; Revised November 9, 2019; Accepted November 11, 2019

*Corresponding author: Sang-Joun Yu, Department of Periodontology, School of Dentistry, Chosun University, 303 Pilmun-daero, Dong-gu, Gwangju 61452, Republic of Korea.

Tel: +82-62-220-3850, Fax: +82-62-224-4664, E-mail: sjyu78@chosun.ac.kr 
The use of the barrier membrane in the GBR technique can increase the amount of regenerated bone [5]. After installation of the dental implant, the use of non-resorbable barrier membrane in the GBR procedure at the dehiscence type defect increases the chances of bone regeneration [6]. However, several limitations exist including the possibility of exposure and the requirement of additional procedure to remove the non-resorbable barrier membrane. Resorbable barrier membranes are preferred due to their high biocompatibility to oral tissues, prevention of bleeding, chemotaxis effect on fibroblast to promote wound healing [7], and no need for their removal. In addition, it was reported that there were no clinical and histological differences between resorbable and non-resorbable barrier membranes when GBR procedure is applied in dehiscence type defect with the same graft material [8].

Furthermore, the probability of bone regeneration in the GBR procedure can be enhanced if coupled with the graft material [9]. In particular, resorbable barrier membrane cannot support the space underneath during the wound healing period due to the lack of rigidity [10]. Therefore, the use of graft material is recommended in order to prevent the collapse of barrier membrane and maintain the space for bone regeneration. Autogenous bone has osteogenic, osteoinductive, and osteoconductive properties and is regarded as the gold standard among various graft materials used with the GBR procedure. However, it is limited in terms of harvesting the graft material and causing discomfort in the donor site. It is possible to overcome these defects by using demineralized freeze dried bone allograft (DFDBA) or hydroxyapatite (HA). DFDBA is expected to play not only an osteoconductive but also an osteoinductive role as the bone morphogenetic protein is exposed during demineralization process [11]. However, DFDBA lacks mechanical rigidity and has a relatively fast resorption rate compared with FDBA or HA. Moreover, it was reported that the amount of bone morphogenetic protein was insufficient to accelerate osteoinduction activity [12]. Several previous studies have reported that HA is capable of maintaining the created space during the healing period and has osteoconductive properties [13]. However, its resorption rate is slow [14]. An alternative approach could be the GBR procedure with FDBA. Indeed, bone formation is faster with cancellous FDBA, but there is a large amount of resorption. On the other hand, cortical FDBA results in slower bone formation, but can maintain the space for a long time [15].

In this study, we aimed to improve the results of the GBR procedure by taking advantage of two graft materials, cancellous and cortical FDBA. Therefore, we applied a 1:1 cancellous:cortical FDBA mixture to treat peri-implant defects with the GBR procedure and compared the results with those of GBR procedure using anorganic bovine bone (ABB) only.

\section{MATERIALS AND METHODS}

\section{Participants}

This randomized controlled clinical trial study was conducted according to the Helsinki Declaration and approved by Chosun University Dental Hospital Ethics Committee (CDMDIRB-1428-167). A total of 37 participants (10 males, 27 females) and 63 sites were evaluated. The mean age of the participants was 55.0 years (range $35-75$ years). Participants were selected from the department of periodontology of Chosun University Dental Hospital.

Each participant agreed to take part in this study and gave written informed consent. Inclusion criteria included participants who had (1) tooth extraction at least 6 months prior to the implant installation, (2) completed initial periodontal treatment including oral hygiene instruction and scaling and root planning, thereby showed good oral hygiene, and (3) dental implants with dehiscence defects related to the installation of implant. Excluded from the study were (1) participants in need of vertical or horizontal GBR procedures before implant installation, (2) participants with a history of long-term therapy with medication that may affect bone healing (oral or IV bisphosphonates, corticosteroids, nonsteroidal anti-inflammatory drugs, etc.), (3) heavy smokers (>10 cigarettes/d), and (4) participants with any medical contraindications for surgery.

\section{Pre-operation}

Prior to the surgery, participants were randomly assigned to one of two groups by a coin flip: (1) control group ( $n=17$, 
32 surgical sites), where ABB material (Bio-Oss ${ }^{\circledR}$; Geistlich Pharma AG, Wolhusen, Switzerland) was used during the application of the GBR procedure to peri-implant defect and (2) experimental group ( $\mathrm{n}=20,31$ surgical sites), where 1:1 cancellous:cortical FDBA allograft mixture (Allo-Oss ${ }^{\circledR}$; CGBio, Seoul, Korea) was used instead of ABB material (Table 1).

Moreover, the inspector was unaware of the randomized process. To obtain acellular plasma, which was mixed with graft material, $10 \mathrm{~mL}$ of blood was drawn from each participant through venipuncture of the left or right arm and placed in sterilized plastic vacuum tubes devoid of silica from their inner surface. Subsequently, blood samples were

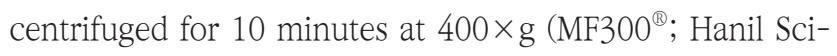
ence Industrial Co., Incheon, Korea). After centrifugation, the supernatant, which consisted of acellular plasma, was aspirated using syringes.

\section{Surgical procedure}

A single inspector carried out the surgical procedure in all participants. After local anesthesia, full-thickness mucoperiosteal flap was reflected. The exposed root and bone surface were debrided with hand and ultrasonic instruments. A surgical template was fabricated based on the restorative treatment plan prior to the surgical procedure. Subsequently, tapered implants were installed in each edentulous site following the manufacturer's recommendations.

\section{Intraoperative measurements}

Once the dehiscence defects were detected on the buccal side of the implants, defect morphology and dimension were measured. Defect morphology was categorized into four types according to the percentage of the implant surface surrounded by bone at the implant shoulder level: (1) no walls, (2) one wall defect $(<33 \%)$, (3) two-wall defects (33\%-67\%), and (4) three-wall defects ( $>67 \%)$. Defect dimension was measured in millimeters using a periodontal probe (CP-12; Hu-Friedy, Chicago, IL, USA). Defect length was measured from the implant shoulder to the base of the defect in the apicocoronal direction. Defect width was calculated between the two points at which the implant and bone were not contacted in the mesial and distal direction at the implant shoulder level (Fig. 1A). Furthermore, two additional points were determined and corresponded to the most outer $\left(D_{i s}\right)$ and inner $\left(D_{d}\right)$ points of the exposed implant surface at the implant shoulder level (Fig. 1B). Intraoral photographs were taken at the buccal and occlusal sides for each participant to corroborate the clinical investigations. In addition, the amount of exposed implant surface was measured. For this calculation, the implants were treated as cylinders with their respective diameters, and the exposed surfaces were treated as curved areas extrapolated onto a plane. Dehisced defects were calculated as half ellipses (Fig. 1C).

\section{Augmentative treatment}

Bones surrounding the procedure site were perforated with a small round bur to induce bleeding from the bone marrows (Fig. 2A, B). Bone transplantation was performed on the surface of the exposed implant. ABB materials were used in the control group, while allograft mixtures comprising cancellous and cortical FDBA in a 50:50 ratio were used in the experimental group. Five minutes prior to the transplantation, acellular plasma, which was obtained by centrifuging blood, was mixed into the graft materials. After bone transplantation, the transplanted site was covered by

Table 1. Number of participants, sex ratio, number of surgical sites, and mean age by group

\begin{tabular}{lccccc}
\hline \multirow{2}{*}{ Group } & No. of participant & \multicolumn{3}{c}{ Sex } & No. of surgical site \\
\cline { 3 - 4 } & 17 & Male & Female & & 32 \\
Control & 20 & 4 & 13 & 31 & 55.65 \\
Experimental & 37 & 6 & 14 & 54.43 & 55.00 \\
Total & 10 & 27 & & 63 \\
\hline
\end{tabular}



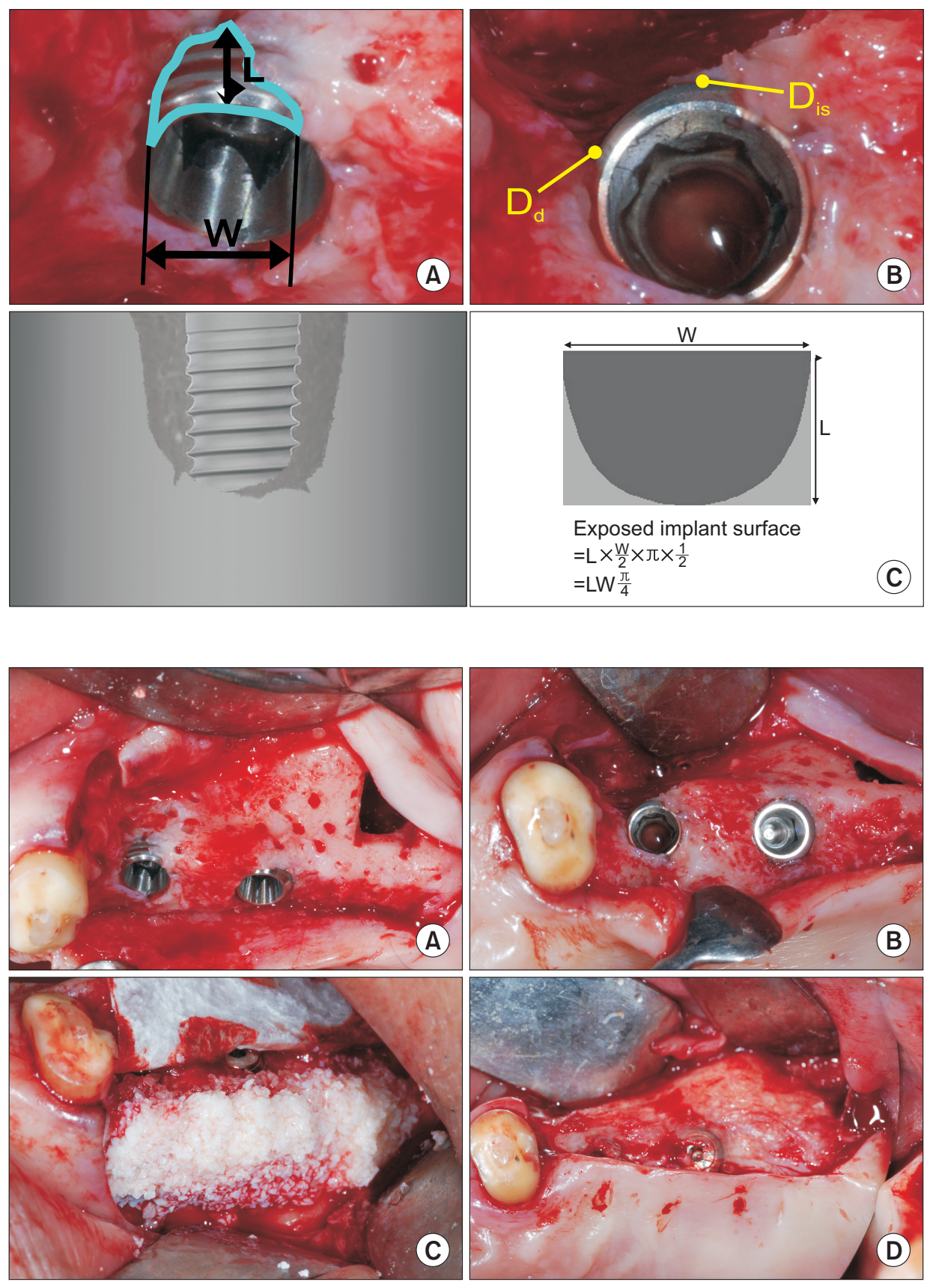

Fig. 1. (A) Measurement of defect length (L) and width (W). (B) The most outer $\left(D_{i s}\right)$ and most inner $\left(D_{d}\right)$ points of the exposed implant surface at the height of implant shoulder level. (C) Formula for calculation of the exposed implant surfaces.

Fig. 2. (A) Exposed implant surface before guided bone regeneration (GBR; buccal view). (B) Exposed implant surface before GBR (occlusal view). (C) GBR was performed on the exposed implant surfaces using the respective graft materials and resorbable barrier membranes in the control and experimental groups. (D) Bone regenerated around the exposed implant surface is visible at re-entry. shaping the collagen barrier membrane with osseoguard ${ }^{\circledR}$ (BIOMET 3i, Warsaw, IN, USA) to extend it at least $2 \mathrm{~mm}$ from the margins of each of the defects (Fig. 2C). During the healing period, Bone Tack ${ }^{\circledR}$ (ACE Surgical Supply Co., Brockton, MA, USA) was applied to increase the stability of the membrane. Periosteal releasing incision was made at the base of the buccal flap to enable coronal repositioning. Furthermore, primary suture was performed with 5-0
Happylon ${ }^{\circledR}$ (Purgo, Sungnam, Korea) non-resorbable suture material to prevent the surgical site from being exposed to the oral cavity.

\section{Postoperative treatment}

After the surgery, antibiotics and information about postoperative care were prescribed and provided equally to 
both the control and experimental groups. Additional drugs were used to control pain when needed. The participants were instructed not to brush the surgical site and instead rinse their mouth for one minute with chlorohexidine $0.2 \%$ twice daily. In addition, they were instructed to use a removable prosthesis after the backing was placed on postoperative day (POD) 10. Sutures were removed over two sessions on POD 7 and 14. The participants visited the hospital 1 and 3 months after the implantation for oral hygiene education and full-mouth scaling. The presence of postoperative complications, such as soft tissue dehiscence, barrier membrane exposure, and implant exposure were also recorded.

\section{Re-entry}

A second implant surgery was performed 5-6 months after the initial implant insertion. A full-thickness flap was reflected for accurate measurement. Bone tack and residual resorbable membrane were removed. Any residual defect length and width were measured with a probe via the same method used initially. Moreover, the amount of regenerated bone toward the buccolingual direction was measured at $D_{i s}$ and $D_{d}$ points using a probe. Pictures of the surgical site were taken at the buccal and occlusal side with an oral camera via the same method used previously (Fig. 2D). Healing abutments were attached instead of using cover screws.

\section{Statistical evaluation}

The extent of reduction of defect length, defect width, and exposed implant surface were calculated by subtracting the re-entry values from the baseline values. The amount of regenerated bone to the buccolingual direction from $D_{i s}$ and $D_{d}$ points was recorded as the amount of horizontal bone gain at $D_{\text {is }}$ and the amount of horizontal bone gain at $D_{\mathrm{d}}$.

Means and standard deviations were calculated for all measurements. A Shapiro-Wilk test of normality was done. t-tests were performed to compare five parameters between the control and experimental groups. The MannWhitney U-test was used to compare the upper and lower measurements between the control and experimental groups. SPSS software ver. 20.0 (IBM Corp., Armonk, NY, USA) was used for all statistical analyses and the level of significance was set at $p<0.05$.

\section{RESULTS}

\section{Defect size at baseline and distribution}

ABB materials were used for GBR on 32 surfaces with exposed implants, while allograft mixtures with 50:50 cancellous FDBA and cortical FDBA were used for 31 surfaces. At baseline, defect length and exposed implant surface were $3.57 \pm 1.44 \mathrm{~mm}$ and $5.83 \pm 5.09 \mathrm{~mm}^{2}$, respectively, in the ABB group, compared with $3.14 \pm 1.23 \mathrm{~mm}$ and $6.98 \pm$ $5.72 \mathrm{~mm}^{2}$, respectively, in the allograft group, with no significant difference between the two groups. On the other hand, the baseline defect width was significantly different between the ABB and allograft groups (ABB: $2.63 \pm 0.92$ mm, allograft: $3.31 \pm 1.22 \mathrm{~mm} ; p<0.05$ ) (Fig. 3).

\section{The extent of reduction of defect size}

There were no significant differences in the amount of reduction of the defect length and exposed implant surface between the ABB $\left(3.47 \pm 1.33 \mathrm{~mm}, 5.53 \pm 4.74 \mathrm{~mm}^{2}\right)$ and allograft $\left(3.10 \pm 1.15 \mathrm{~mm}, 6.69 \pm 5.46 \mathrm{~mm}^{2}\right)$ groups. On the other hand, there was a significant difference in

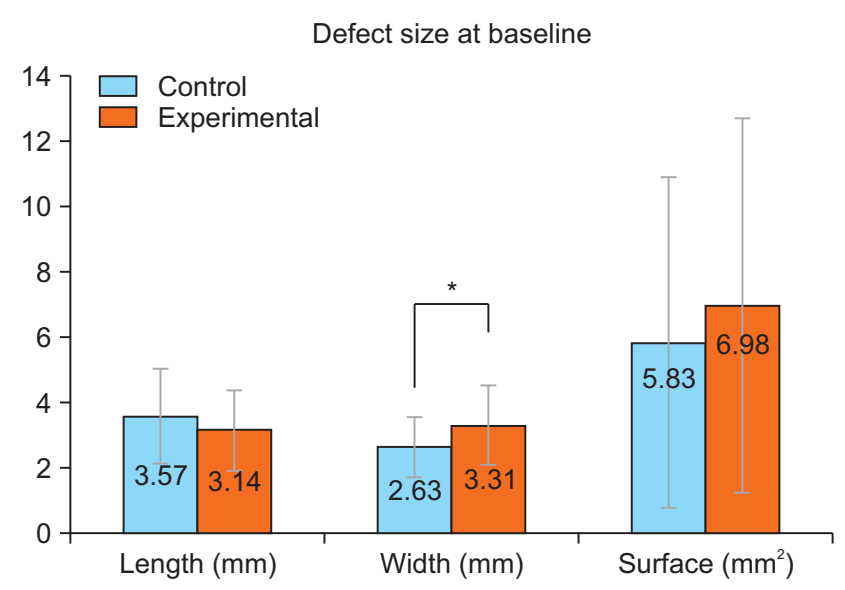

Fig. 3. Defect length, width, and exposed implant surface of control and experimental groups at baseline. ${ }^{\star}$ Statistically significant difference $(p<0.05)$. 
the amount of reduction of defect width between the $\mathrm{ABB}$ $(2.58 \pm 0.89 \mathrm{~mm})$ and allograft $(3.19 \pm 1.27 \mathrm{~mm})$ groups $(p$ $<0.05)$ (Fig. 4). The amounts of horizontal bone gain from $D_{\text {is }}$ and $D_{d}$ points were $1.68 \pm 0.46 \mathrm{~mm}$ and $3.28 \pm 1.56 \mathrm{~mm}$, respectively, in the $\mathrm{ABB}$ group and $1.74 \pm 0.56 \mathrm{~mm}$ and 2.95 $\pm 1.45 \mathrm{~mm}$, respectively, in the allograft; but no significant difference were observed between the two groups (Fig. 5).

\section{Comparison of upper and lower measurements between the control and experimental groups}

At baseline, the control and experimental groups did not significantly differ in upper and lower defect length, defect width, and exposed implant surface. Furthermore, the amount of reduction of defect length, defect width, exposed implant surface in addition to the amount of horizontal bone gain at $D_{\text {is }}$ and $D_{d}$ points all did not show statistically significant difference between $\mathrm{ABB}$ and allograft groups (Table 2).

\section{Defect morphology}

A two-wall and three-wall osseous defect accounted for $21.9 \%$ and $75.0 \%$, respectively, in the control group and $35.5 \%$ and $61.3 \%$, respectively, in the experimental group. In other words, most of the participants in the control and experimental groups had either a two-wall or three-wall osseous defect. However, compared with the controls, the

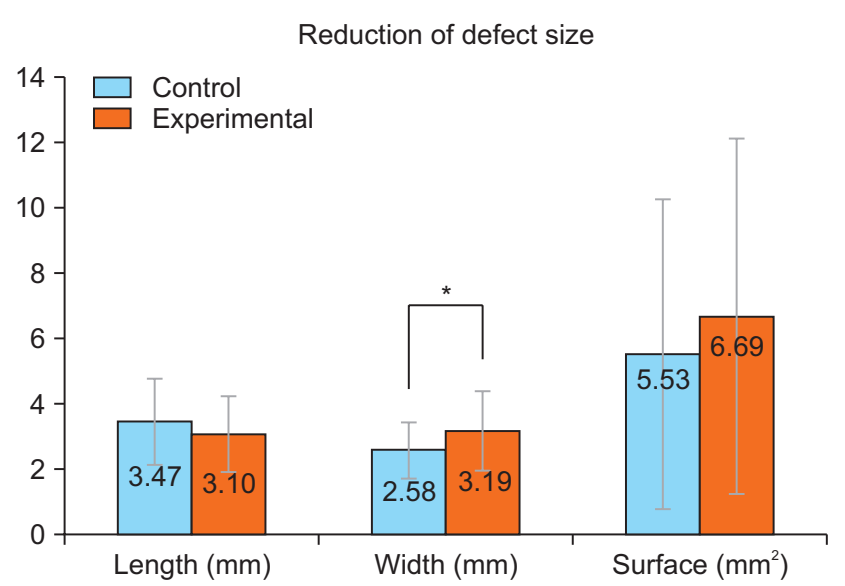

Fig. 4. Reduction of defect length, width, and exposed implant surface of control and experimental groups. ${ }^{*}$ Statistically significant difference $(p<0.05)$.

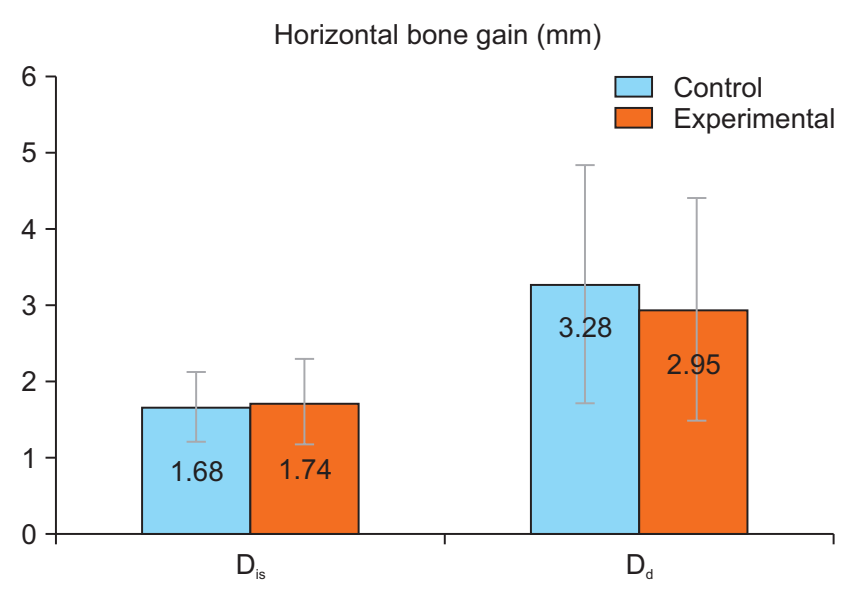

Fig. 5. Horizontal bone gain at the most outer $\left(D_{i s}\right)$ and most inner $\left(D_{d}\right)$ points of the exposed implant surface at the height of implant shoulder level.

Table 2. Defect length, width, and exposed implant surface at baseline and re-entry and horizontal bone gain at $D_{i s}$ and $D_{d}$ measurement points in maxilla and mandible

\begin{tabular}{|c|c|c|c|c|c|c|c|c|c|c|c|}
\hline & \multicolumn{3}{|c|}{ Length (mm) } & \multicolumn{3}{|c|}{ Width (mm) } & \multicolumn{3}{|c|}{$\begin{array}{l}\text { Exposed implant } \\
\text { surface }\left(\mathrm{mm}^{2}\right)\end{array}$} & \multicolumn{2}{|c|}{$\begin{array}{l}\text { Horizontal bone } \\
\text { gain }(\mathrm{mm})\end{array}$} \\
\hline & Base-line & Re-entry & $\Delta$ & Base-line & Re-entry & $\Delta$ & Base-line & Re-entry & $\Delta$ & $D_{\text {is }}$ & $\mathrm{D}_{\mathrm{d}}$ \\
\hline \multicolumn{12}{|l|}{ Maxilla } \\
\hline Control & $3.39 \pm 1.12$ & $0.06 \pm 0.03$ & $3.34 \pm 1.08$ & $2.64 \pm 0.99$ & $0.01 \pm 0.00$ & $2.63 \pm 0.99$ & $5.23 \pm 4.52$ & $0.00 \pm 0.00$ & $5.07 \pm 4.31$ & $1.61 \pm 0.64$ & $3.30 \pm 1.38$ \\
\hline Experimental & $3.42 \pm 1.27$ & $0.08 \pm 0.02$ & $3.36 \pm 1.15$ & $3.50 \pm 1.65$ & $0.15 \pm 0.05$ & $3.35 \pm 1.56$ & $7.38 \pm 4.94$ & $0.02 \pm 0.01$ & $6.88 \pm 4.86$ & $1.42 \pm 0.63$ & $3.43 \pm 1.78$ \\
\hline \multicolumn{12}{|l|}{ Mandible } \\
\hline Control & $3.60 \pm 1.51$ & $0.11 \pm 0.05$ & $3.50 \pm 1.33$ & $2.62 \pm 0.88$ & $0.08 \pm 0.03$ & $2.53 \pm 0.81$ & $6.35 \pm 4.79$ & $0.03 \pm 0.01$ & $5.94 \pm 4.31$ & $1.74 \pm 0.28$ & $3.28 \pm 1.74$ \\
\hline Experimental & $3.07 \pm 1.31$ & $0.03 \pm 0.02$ & $3.03 \pm 1.20$ & $3.22 \pm 0.98$ & $0.11 \pm 0.05$ & $3.10 \pm 0.90$ & $6.81 \pm 5.14$ & $0.00 \pm 0.00$ & $6.60 \pm 4.82$ & $1.89 \pm 0.47$ & $2.71 \pm 1.24$ \\
\hline
\end{tabular}

Values are presented as mean \pm standard deviation.

$\mathrm{D}_{\mathrm{i}}$, the most buccal points of the exposed implant surface at the implant shoulder level; $\mathrm{D}_{\mathrm{d}}$, the most lingual points of the exposed implant surface at the implant shoulder level; $\Delta$, base-line - re-entry. 
Table 3. Proportion of 1-, 2-, and 3-wall defect types in the control and experimental groups

\begin{tabular}{lccc}
\hline \multirow{2}{*}{ Group } & \multicolumn{3}{c}{ Defect type } \\
\cline { 2 - 4 } & 1-wall & 2-wall & 3-wall \\
\hline Control $(\mathrm{n}=32)$ & $1(3.1)$ & $7(21.9)$ & $24(75.0)$ \\
Experimental $(\mathrm{n}=31)$ & $1(3.2)$ & $11(35.5)$ & $19(61.3)$ \\
\hline
\end{tabular}

Values are presented as number (\%).

experimental group had a lower proportion of three-wall osseous defect, but a higher proportion of a two-wall osseous defect (Table 3).

\section{Post-guided bone regeneration complications}

During the 5-6 months healing period between GBR and re-entry, there were no problems such as soft tissue dehiscence, barrier membrane exposure, and implant exposure in both control and experimental groups.

\section{DISCUSSION}

In this study, GBR was performed either with $\mathrm{ABB}$ or allograft mixture (50:50 cancellous FDBA and cortical FDBA) with collagen barrier membranes on exposed implant surfaces. The results revealed that a high level of new bone formation was obtained in both the control (ABB) and experimental (allograft) groups, as previously documented. Several studies have performed GBR on exposed implant surfaces using collagen barrier membranes and ABB. Zitzmann et al. [16] performed GBR on 39 sites and reported a 92\% reduction of exposed implant surface (from $15.8 \pm 9.52$ to $1.1 \pm 2.84 \mathrm{~mm}$ ) at re-entry 4-6 months after GBR. Nemcovsky et al. [17] performed GBR on 21 patients (28 sites) and reported a 91\% reduction of defect length (6.7 \pm 2.23 to $0.6 \pm 0.69 \mathrm{~mm}$ ), an $84 \%$ reduction of defect width ( $4.3 \pm 0.90$ to $0.7 \pm 0.77 \mathrm{~mm}$ ), and a $97 \%$ reduction of $\mathrm{ex}^{-}$ posed implant surface $(23.7 \pm 11.49$ to $0.7 \pm 0.99 \mathrm{~mm})$, after 6-8 months. After performing GBR on 10 sites, Hämmerle and Lang [18] reported a 31\% reduction of defect length (3.6 \pm 1.6 to $2.5 \pm 0.6 \mathrm{~mm}$ ), a $100 \%$ reduction of defect width $(0.5 \pm 0.5$ to $0 \pm 0 \mathrm{~mm})$, and an $86 \%$ reduction of exposed implant surface, after 6-7 months. On the other hand, some studies performed GBR on exposed implant surfaces using collagen barrier membranes and allograft mixtures with cancellous and cortical FDBA. For instance, Park et al. [19] used mineralized human cancellous allograft as the inner layer and mineralized human cortical allograft as the outer layer. In the same study, the amount of reduction of defect length and exposed implant surface were $68.14 \%$ and $78.73 \%$, respectively.

To date, most studies have measured the amount of reduction of defect length, width, and exposed implant surface to assess bone regeneration after performing GBR on exposed implant surfaces [20]. Measuring these parameters may be meaningful in determining the degree of improvement in cases in which a near 100\% bone regeneration was not achieved around the exposed implant surfaces. However, the amount of reduction of exposed implant surface was close to $100 \%$ in both the control and experimental groups in this study. Moreover, the shape of the bone surrounding the implant becomes the base of soft tissues superior to the ridge crest [21]. Because soft tissues determine the aesthetic outcome [22], bone shape is one of the determinants of an aesthetic soft tissue shape. Thus, we measured here the amount of horizontal bone gain from $\mathrm{D}_{\mathrm{is}}$ and $\mathrm{D}_{\mathrm{d}}$ points in addition to the amount of reduction of defect length, width, and exposed implant surface, thus increasing the value of our present study. To date, only one study has measured the amount of horizontal bone gain after performing GBR on exposed implant surfaces [19]. In that study, authors performed GBR with mineralized human cancellous allograft (inner layer), mineralized human cortical allograft (outer layer), and bovine collagen membrane on nine sites and reported the amount of horizontal bone gain at the most lingual point to be $1.57 \pm 0.76 \mathrm{~mm}$. The discrepancy to our findings may be attributable to a few reasons. First, while Park et al. limited the initial graft thickness to $3 \mathrm{~mm}$ horizontally using a stent during GBR [19], we did not limit the graft material. Second, five out of nine patients had barrier membrane or implant exposure in the previous study, but no complications were observed both in the control and experimental group in our study. Complications such as flap dehiscence may induce infection and inflammation of the tissue inferior to or surrounding the barrier membrane [23], and breakdown of collagen barrier membranes caused by bacterial collagenases leads 
to insufficient bone regeneration [24]. Indeed, many studies have examined the relation between incidence of complications and degree of inadequate bone regeneration. Zitzmann et al. [16] performed implantation and GBR using ABB materials and resorbable barrier membranes simultaneously and reported wound dehiscence in seven out of 43 sites (16\%) with an $87 \%$ reduction of exposed implant surface. On the other hand, improvements reached $94 \%$ in cases that did not involve complications. Hämmerle and Lang [18] reported that complications developed in two out of 10 sites (20\%). In the eight remaining sites without complications, the amount of reduction of exposed implant surface was $100 \%$. The amount of reduction of exposed implant surface was $60 \%$ in one site with superficial soft tissue necrosis and $0 \%$ in the remaining site with signs of marginal inflammation of the mucosa and infection of the site during the healing phase. Furthermore, Park et al. [19] reported that the amount of horizontal bone gain in only the sites without barrier membrane or implant exposure was 1.92 $\pm 0.52 \mathrm{~mm}$, showing that bone regeneration considerably increased in sites without complications. As shown here, GBR is a sensitive procedure, in which the amount of bone regeneration is influenced by the skills of the surgeon and the resulting development of complications. Therefore, in this study, one skilled surgeon with vast experience performed all the surgeries.

GBR in 2-wall osseous defects is less predictable than 3-wall osseous defects, and defects are less likely to favor bone growth over exposed implant surface. Zitzmann et al. [16] performed GBR with resorbable membranes and ABB mineral in 2- and 3-wall osseous defects and reported a $92 \%$ reduction of exposed implant surface. In the present study, despite the fact that the proportion of 3 -wall osseous defect was lower while that of 2-wall osseous defect was higher in the experimental group, there was no significant difference between the control and experimental groups in the amount of reduction of defect width. Moreover, it is difficult to conclude that the control and experimental groups significantly differed in the amount of bone regeneration, as both groups significantly differed in defect width at baseline.

In this study, cancellous and cortical FDBA were mixed in a 50:50 ratio. The primary reason is related to the bone graft repair process in cancellous and cortical bones [15]. Because revascularization occurs at a more rapid rate in cancellous bones, bone formation occurs before bone resorption by osteoclasts, resulting in a quick and complete replacement of old bones with new bones. This process is referred to as "creeping substitution," where quick resorption of graft materials promote bone to implant contact and ensure osseointegration. On the other hand, revascularization occurs at a slow rate in cortical bones, which have a limited number of endosteal cells that are involved in vascular anastomosis [25]. Bone resorption occurs first, widening the haversian cavity, and bone formation follows only after an adequate amount of osteoclast activitya process called "reverse creeping substitution." Therefore, cortical bones do not tend to heal completely with time, but rather remain a mixture of necrotic and vital bones. These properties allow cortical bones to hold the space for a longer period of time. In essence, cancellous FDBA is not only associated with rapid bone formation but also results in large resorption of graft material. In contrast, cortical FDBA involves slow bone formation, but may maintain space for a long period. We mixed cancellous and cortical FDBA in 50:50 ratio in an attempt to utilize the merits of the two types of grafts and improve the outcomes of GBA on exposed implant surfaces.

In this study, acellular plasma, which was obtained by centrifuging the patient's blood, was mixed with the grafts before placing the grafts on the defect area. If the patient's blood is centrifuged in a sterilized plastic vacuum tube not containing silica, two layers are formed with the red blood cells at the bottom layer and plasma layer on top. Mixing acellular plasma with bone graft materials agglutinates the material, making it easier for the surgeon to place the graft materials on the defect area [26].

Dimensional changes of the ridge crest still occur even after measurement at re-entry after GBR [27]. In this study, we waited for about 5-6 months for healing, but this was not enough for a complete remodeling of the bone grafts. Furthermore, the degree of bone formation in the defect area after implant insertion was visually evident at the second implant surgery. However, there have been no histological analyses of regenerated tissues or implant-bone interface. 
The amount of horizontal bone gain in our study has clinical relevance in implant therapy. Exposure of implant thread is irrelevant to problems in the surrounding mucus or progressive bone resorption [28], but stable vertical dimension by ensuring a sufficient horizontal bone thickness is considered essential for aesthetics. However, there has been no long-term study that investigated the relationship between horizontal bone thickness obtained through simultaneous implant insertion and GBR and the stability of the labial and buccal bone height. We plan to examine this relationship through a long-term study at a later opportunity. In addition, based on the maximum distance between the exposed implant surface and buccal wall, we also aim to investigate the extent and amount of bone regeneration for each distance stimulated by GBR using allografts and ABBs in the future.

Within the limits of this study, this study revealed that GBR is effective on exposed implant surfaces following the initial implant insertion. Using allografts comprising 50:50 cancellous and cortical FDBA in addition to resorbable barrier membranes during the GBR procedure is effective, and advances an alternative for GBR with ABB materials.

\section{ACKNOWLEDGEMENTS}

This study was supported by the research fund from Chosun University Dental Hospital, 2017.

\section{CONFLICTS OF INTEREST}

The authors declare that they have no competing interests.

\section{ORCID}

\author{
Won-Pyo Lee \\ https://orcid.org/0000-0003-1911-3454 \\ Do-Young Park \\ https://orcid.org/0000-0003-4724-0274 \\ Ki-Won Lee \\ https://orcid.org/0000-0002-5168-9560 \\ Keon-Il Yang \\ https://orcid.org/0000-0001-8789-7743
}

Byung-Ock Kim

https://orcid.org/0000-0001-8952-617X

Sang-Joun Yu

https://orcid.org/0000-0001-8818-549X

\section{REFERENCES}

1. Chiapasco M, Zaniboni M. Clinical outcomes of GBR procedures to correct peri-implant dehiscences and fenestrations: a systematic review. Clin Oral Implants Res 2009;20 Suppl 4:113-123. doi: 10.1111/j.1600-0501.2009.01781.x.

2. Kopp KC, Koslow AH, Abdo OS. Predictable implant placement with a diagnostic/surgical template and advanced radiographic imaging. J Prosthet Dent 2003;89:611-615. doi: 10.1016/s0022-3913(03)00198-7.

3. Wang HL, Al-Shammari K. HVC ridge deficiency classification: a therapeutically oriented classification. Int J Periodontics Restorative Dent 2002;22:335-343.

4. Rutkowski JL. Vertical alveolar ridge augmentation in implant dentistry: a surgical manual and horizontal alveolar ridge augmentation in implant dentistry: a surgical manual. Tolstunov L, ed. Hoboken, NJ: John Wiley \& Sons, Inc. Hoboken, New Jersey. J Oral Implantol 2016;42:518. doi: 10.1563/aaid-joi-D-Review.4206.

5. Jung SH, Chang HY, You HK, Pi SH. Clinical effect of porous titanium mesh with cross-linked collagen membrane for guided bone regeneration. Oral Biol Res 2019;43:189195. doi: 10.21851/obr.43.03.201909.189.

6. Dahlin C, Lekholm U, Becker W, Becker B, Higuchi K, Callens A, van Steenberghe D. Treatment of fenestration and dehiscence bone defects around oral implants using the guided tissue regeneration technique: a prospective multicenter study. Int J Oral Maxillofac Implants 1995;10:312318.

7. Postlethwaite AE, Seyer JM, Kang AH. Chemotactic attraction of human fibroblasts to type I, II, and III collagens and collagen-derived peptides. Proc Natl Acad Sci U S A 1978;75:871-875. doi: 10.1073/pnas.75.2.871.

8. Oh TJ, Meraw SJ, Lee EJ, Giannobile WV, Wang HL. Comparative analysis of collagen membranes for the treatment of implant dehiscence defects. Clin Oral Implants Res 2003;14:80-90. doi: 10.1034/j.1600-0501.2003.140111.x.

9. Gelb DA. Immediate implant surgery: three-year retrospective evaluation of 50 consecutive cases. Int J Oral Maxillofac Implants 1993;8:388-399.

10. Jovanovic SA, Spiekermann H, Richter EJ. Bone regeneration around titanium dental implants in dehisced defect sites: a clinical study. Int J Oral Maxillofac Implants 1992;7:233-245.

11. Urist MR, Iwata H. Preservation and biodegradation of the morphogenetic property of bone matrix. J Theor Biol 1973;38:155-167. doi: 10.1016/0022-5193(73)90231-2. 
12. Tsai CH, Chou MY, Jonas M, Tien YT, Chi EY. A composite graft material containing bone particles and collagen in osteoinduction in mouse. J Biomed Mater Res 2002;63:6570. doi: 10.1002/jbm.10089.

13. Fugazzotto PA. GBR using bovine bone matrix and resorbable and nonresorbable membranes. Part 1: histologic results. Int J Periodontics Restorative Dent 2003;23:361-369. doi: $10.11607 /$ prd.00.0532.

14. Skoglund A, Hising P, Young C. A clinical and histologic examination in humans of the osseous response to implanted natural bone mineral. Int J Oral Maxillofac Implants 1997;12:194-199.

15. Burchardt H. The biology of bone graft repair. Clin Orthop Relat Res 1983;(174):28-42. doi: 10.1097/00003086198304000-00005.

16. Zitzmann NU, Naef R, Schärer P. Resorbable versus nonresorbable membranes in combination with Bio-Oss for guided bone regeneration. Int J Oral Maxillofac Implants 1997;12:844-852.

17. Nemcovsky CE, Artzi Z, Moses O, Gelernter I. Healing of dehiscence defects at delayed-immediate implant sites primarily closed by a rotated palatal flap following extraction. Int J Oral Maxillofac Implants 2000;15:550-558.

18. Hämmerle CH, Lang NP. Single stage surgery combining transmucosal implant placement with guided bone regeneration and bioresorbable materials. Clin Oral Implants Res 2001;12:9-18. doi: 10.1034/j.1600-0501.2001.012001009. $\mathrm{x}$.

19. Park SH, Lee KW, Oh TJ, Misch CE, Shotwell J, Wang HL. Effect of absorbable membranes on sandwich bone augmentation. Clin Oral Implants Res 2008;19:32-41. doi: 10.1111/j.1600-0501.2007.01408.x.

20. Moses O, Pitaru S, Artzi Z, Nemcovsky CE. Healing of dehiscence-type defects in implants placed together with different barrier membranes: a comparative clinical study.
Clin Oral Implants Res 2005;16:210-219. doi: 10.1111/ j.1600-0501.2004.01100.x.

21. Burkhardt R, Joss A, Lang NP. Soft tissue dehiscence coverage around endosseous implants: a prospective cohort study. Clin Oral Implants Res 2008;19:451-457. doi: 10.1111/j.1600-0501.2007.01497.x.

22. Linkevicius T, Apse P. Biologic width around implants. An evidence-based review. Stomatologija 2008;10:27-35.

23. Nowzari H, Slots J. Microbiologic and clinical study of polytetrafluoroethylene membranes for guided bone regeneration around implants. Int J Oral Maxillofac Implants 1995;10:67-73.

24. Donos N, Kostopoulos L, Karring T. Alveolar ridge augmentation using a resorbable copolymer membrane and autogenous bone grafts. An experimental study in the rat. Clin Oral Implants Res 2002;13:203-213. doi: 10.1034/ j.1600-0501.2002.130211.x.

25. Enneking WF, Burchardt H, Puhl JJ, Piotrowski G. Physical and biological aspects of repair in dog cortical-bone transplants. J Bone Joint Surg Am 1975;57:237-252.

26. Kim JS, Jeong MH, Jo JH, Kim SG, Oh JS. Clinical application of platelet-rich fibrin by the application of the Double $\mathrm{J}$ technique during implant placement in alveolar bone defect areas: case reports. Implant Dent 2013;22:244-249. doi: 10.1097/ID.0b013e3182920da3.

27. Chiapasco M, Romeo E, Casentini P, Rimondini L. Alveolar distraction osteogenesis vs. vertical guided bone regeneration for the correction of vertically deficient edentulous ridges: a 1-3-year prospective study on humans. Clin Oral Implants Res 2004;15:82-95. doi: 10.1111/j.16000501.2004.00999.x.

28. Lekholm U, Sennerby L, Roos J, Becker W. Soft tissue and marginal bone conditions at osseointegrated implants that have exposed threads. A 5-year retrospective study. Int J Oral Maxillofac Implants 1996;11:599-604. 